\title{
Internet-technology for Remote User Support OPTCON
}

\author{
Liudmila Massel ${ }^{1}$, Alexander Gornov ${ }^{2}$, Tatiana Zarodnyuk ${ }^{3}$ \\ ${ }^{1}$ Melentiev Energy Systems Institute of Siberian Branch of the Russian Academy of Sciences, \\ Lermontov str., 130 \\ Irkutsk, Russia \\ E-mail: massel@isem.irk.ru
}

${ }^{2}$ Matrosov Institute for System Dynamics and Control Theory of Siberian Branch of the Russian Academy of Sciences, Lermontov str., 134 Irkutsk, Russia

E-mail: gornov@icc.ru

\author{
${ }^{3}$ Matrosov Institute for System Dynamics and Control Theory of Siberian Branch of the Russian Academy of Sciences, \\ Lermontov str., 134 \\ Irkutsk, Russia \\ E-mail:tz@icc.ru
}

\begin{abstract}
The paper presents a methodical approach to the construction of modern software systems, as well as the creation of computing resources accessible via the Internet. The developed technologies are applied to solve the problem of legacy software using the example of the software OPTCON for solving optimal control problems.

Keywords: Software, mathematical model, computational experiment.
\end{abstract}

\section{Introduction}

The application of mathematical methods in scientific and applied research is significantly hampered by the weakness of the technologies for generating models. The creation of a mathematical model of the phenomenon requires, as a rule, considerable time and effort of a large team, which includes specialists in the subject area, mathematical modeling specialists and specialists in computational algorithms [1]. The paper proposes an approach to the creation and numerical study of mathematical models of controlled processes described by systems of ordinary differential equations, based on Internet technologies [2]-[4] and OPTCON optimization programs [5]-[7].

The development of Internet technology for remote user support OPTCON is aimed at creating a new information technology for mathematical modeling and computational experiments using the OPTCON-2 software for solving problems arising in various areas of fundamental and applied research: energetics, economy, ecology, flight dynamics, navigation, robotics, chemical kinetics, sociology, etc. [2, 8].
The need for such a development is very large, as with the complexity of different branches of knowledge, the need for solving optimal control problems increases. At the same time, the complexity of mathematical methods has grown so much that for a non-specialist in mathematics their use is fraught with various difficulties. For example, at the present time in the corporation Boeing, under the leadership of J. Betts, a similar software package for optimal control of SOCS (Spare Optimal Control Sortware) is being developed [9], [10].

In addition, it is assumes an effective solution to the problem of supporting the researcher on the part of mathematicians throughout the entire computing experiment. User support is necessary because in solving the problem of optimal control we need to take into account a multitude of factors related to the subject area, the mathematical side of the problem, and the peculiarities of the software for solving these problems. Thus, the use of software to solve the optimal control problem is impossible without the participation of a mathematician, and often without a PC developer.

The purpose of the work at this stage is to identify requirements for the functionality of the system, design 
its main components and develop system-conceptual agreements.

\section{Scenarios for applying the of mathematical modeling method}

We can distinguish the following typical scenarios that arise when trying to apply the mathematical modeling method for the practical study of controlled processes:

(i) The researcher has a clearly formulated problem of optimal control.

(ii) The researcher has a parametrized model of the phenomenon dynamics, constructed deductively on the basis of general laws.

(iii) The researcher has numerous information and facts about the process being studied in an informal way - measurement tables, graphs, expert estimates of the mutual influence of factors.

In the first case, which is quite rare in practice, the problem lies in the correct setting of the problem for the software package, monitoring the progress of the calculations, evaluating the quality of the solution obtained. The properties of modern optimization algorithms do not provide a guaranteed solution in all cases, however, using the standard multimethod schemes of software application [5], the probability of achieving success is quite high, especially for smallscale problems.

In the absence of a "personified model", but with a known structure of the differential equations system, the first stage of the solution may be to select the coefficients that allow one to find the system version most closely matches the available experimental data. This parametric identification problem is in the search for the proximity criterion (usually the least-squares functionals) and the solution of a special problem of mathematical programming.

With a satisfactory solution of the problem of the first stage, the problem reduces to first step, and the second stage of the solution consists in the correct application of the optimization pact.

In the third most common case, in the absence of an expert in modeling, the application of mathematical approaches is reduced, as a rule, to the use of statistical methods that have limited possibilities of extracting information from data and do not allow the use of the control theory results.

\subsection{Technologies of interaction between the user and the expert.}

In complex situations that arise for the user in solving the problem, the expert is involved in one of the offline or online schemes. The offline scheme assumes the fixation of calculations by the user in the database on the server and access to the expert via e-mail. The expert, having studied the current situation, suggests ways of overcoming difficulties and sends a letter with his suggestions.

Interaction with the online scheme is realized through the technology of direct communication between the user and the expert through the Internet. The user and the expert, having agreed in advance about the time of the synchronous session, load simultaneously their versions of the interface and solve the problem together in real time.

\subsection{A computational experiment ideology}

In the course of the study of the problem, it was revealed that the technology of carrying out the computational experiment has an iterative character and includes the following main stages:

(iv) Verbal description of the problem.

(v) Mathematical statement of the problem

(vi) The solution of the problem.

(vii) Analysis of the results.

(viii) Adjustment of the problem.

(ix) Interpretation of the solution.

(x) Selecting template actions. Major Headings.

Major headings should be typeset in boldface with the first letter of important words capitalized.

At the first stage, the researcher describes the problem in terms of the domain, produces the selection of entities and relationships between them, describes the dynamics of changes in various parameters.

After the verbal description, the formulation of the mathematical problem is performed, the researcher together with the specialist produce the formulation of the optimal control problem, set the initial conditions, etc.

The solution of the problem is carried out with the help of PC OPTCON and can be performed: both in batch and in interactive mode. And part of the problem can be solved in batch mode, and when some event occurs, for example, if some value exceeds the limits of the definition area, the calculation procedure stops and, after waiting for the user's instructions, switches to interactive mode.

When obtaining a solution that does not meet the necessary criteria, the problem parameters are adjusted. If a "good" solution is received, then it is interpreted in terms of the subject domain.

Throughout the entire computing experiment, from the verbal formulation of the problem and until the final 
decision is reached, the researcher receives all the necessary support from the specialist On the basis of a number of experiments, template actions can be singled out, which in the future will be used to solve the corresponding problems without the support of a specialist.

\section{General technological scheme and its components}

The proposed technology (Fig. 1.) allows to use different scenarios of interaction between the user, the expert and the optimization package depending on the user's awareness and model readiness (the first, the second and the third scenarios).

At each stage of the solution, different languages (L1, L2, L3 and L4) are used to adequately represent the required information.

A content problem statement is a description of the problem in the L1-language, the most remote from mathematical and program terms. The main concepts of the L1-language are factors, quantities that vary with

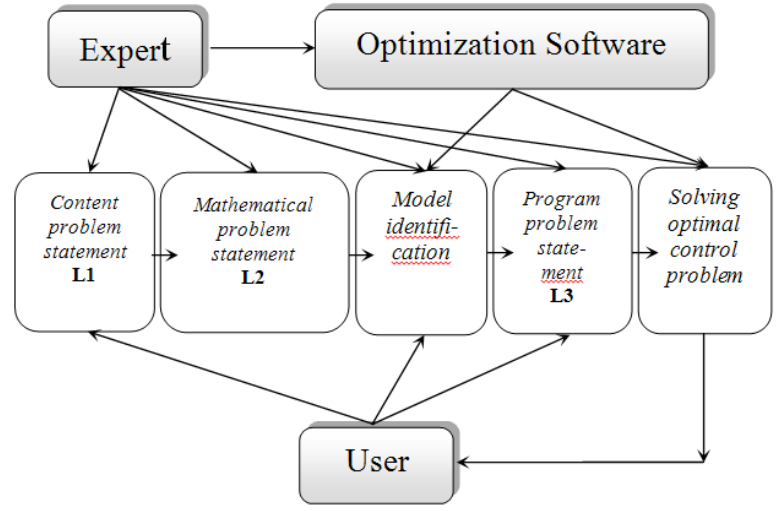

Fig. 1. Technological scheme of setting and solving the optimal control problem.

time, and constants. The user selects the significant factors that are taken into account in its subject area, divides them into simulated and non-simulated. The user sets the tabular or graphic of the data changes in these factors.

Further, in terms of factors, the user generates control objectives (the aim functionals and constraints) and selects the class of applied models - linearquadratic, polynomial, fractional-rational or nonlinear models.

According to the user-defined data, the identification problems for model parameters are generated, after solving which the problem can be automatically written in the L3-language (Program problem statement).

The Mathematical problem statement, carried out in the L2-language, is closest in appearance to the usual mathematical notations. Basic terms are phase variable, control, time interval, initial state, dimensions, parallelepiped constraint, terminal and phase constraint, objective functional. The same language describes the requirements for an approximate solution (the accuracy of integration, the accuracy of the optimal criteria, the step of discretization). The translation of the problem statement from the L2-language to the L3-language is also automatic.

"Personification of the problem" - the selection of a set of controlled systems of a particular model, most appropriate to the user's existing views and experimental data, is performed by solving parametric identification problems. The optimized variables are the parameters of the model, the aim functional is one of the least-squares closeness criteria.

The Program problem statement is the recording of all the components of the statement in the input L3language of the optimization software (in our case OPTCON-2, this is a subset of the $\mathrm{C}$ programming language). Next, using the standard $\mathrm{C}$ compiler, the problem statement is compiled and combined with the software.

\section{Description of the software}

OPTCON-2 is implemented as a software of three-tier architecture "client-server". The structure of the software is shown in Fig. 2.

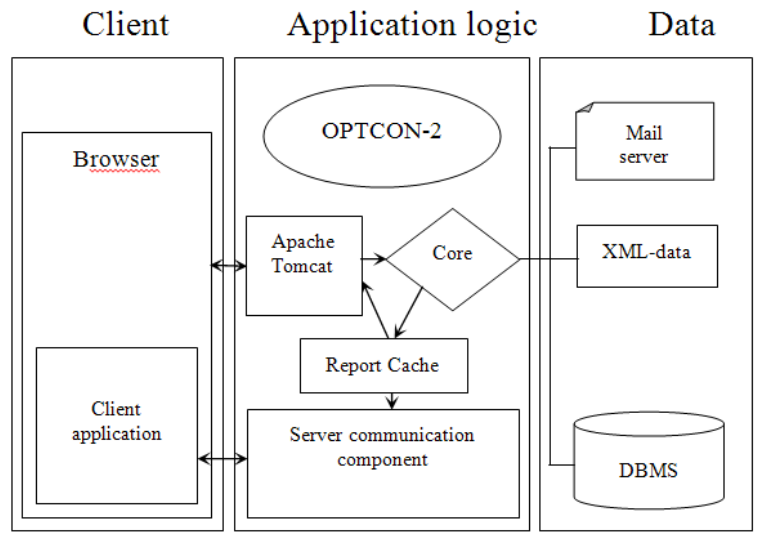

Fig. 2. Software architecture. 


\subsection{The server software}

The following components are includes in the server software:

- The Linux operating system or, perhaps, some other one, which in due course will satisfy the existing needs.

- Apache-Tomcat Web-server for dynamic creation of HTML-pages and organization of data exchange with the client's browser.

- The computing core of ORTCON-2, which provides the solution of the optimal control problem.

- Database for data management.

- The server part of the communication application for data exchange with the client application

- Cache for data accumulation.

- Server core, providing the joint work of all components

The server side software may also include a mail server for sending notifications to users and other components.

\subsection{Customer software}

The client-side software includes:

- Internet browser.

- The client part of the communication application.

The client part of the communication application is an Active-X component or a Java applet. Its use is due to the large amount of data that the client and the server are constantly exchanging, and the user's little response time to this data. When using the client application, the functionality of the client part also increases. Using only a browser for this purpose will not be justified.

\subsection{The technology of the software}

When the client accesses the server, authorization and installation of the HTTP session occurs, after which the server sends a response to the client, including the set from the HTML data and data for the client application. After the client application is initialized, the connection between the client application and the server part is established in the browser. If communication between the client and the server is lost, then all messages accumulate in the cache and after the session is restored are sent to the client. Such a data exchange technology increases the reliability of data security and allows for: loss of communication or disconnection from the computing server, do not stop the calculation, and upon restoring communication receive all the accumulated messages from the system. On the other hand, with incorrect use of this technology, calculations may appear; not needed for the user and not completed by him. To track such calculations, you can set the socalled "time to live (TTL) for calculation, which is prolonged after each treatment the user to this calculation. If during this time there was not a single connection, a warning is sent to the user, this calculation is "frozen" and stored for a while, after which if it was not accessed, it is canceled.

At work the researcher and the specialist can jointly make decisions regarding the problem. The researcher can receive consultation from a specialist in real time or in the offline mode session. The interaction of the software of the researcher and specialist is built on the technology of network interaction between computers peer-to-peer (equal to equal) [11].

The main idea of this technology is decentralization (Fig. 3.) of computers on the network to increase the speed of information exchange. Using peer-to-peer technology will not make a difference in the handling of requests from researchers and professionals, as well as give an opportunity to lower the system requirements on the client side and to organize such a mode, in which for one problem at a time will be able to operate any number of researchers and experts.

For data transfer, HTTP or S-HTTP protocols are used that allow to transfer HTML data to the browser, and a protocol based on UDP sockets for data exchange with the client application.

The use of the UDP protocol is justified by the high rate of "birth" and "obsolescence" of information, so the use of protocols based on the TOP will not be justified because of the need for additional confirmation of the correctness of the data [12].

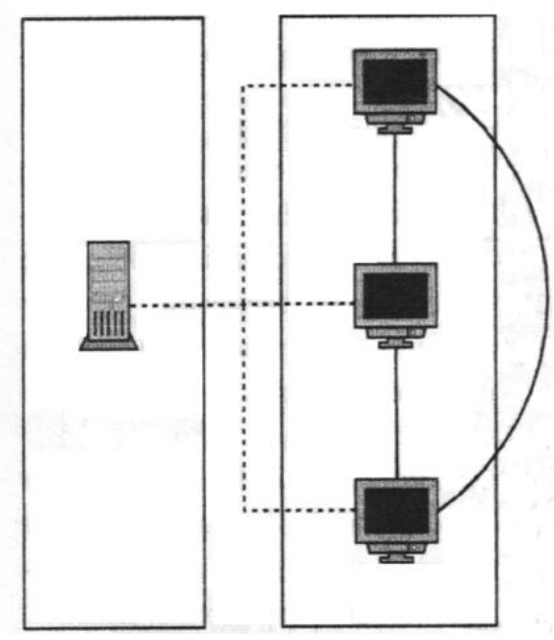

Fig. 3. Technologies of network interaction of computers peer-to-peer. 
However, there is a problem in creating a secure connection between the client application and the server. She is can be solved with the help of additional encryption of traffic by crypto algorithms, based on public keys

\section{Conclusions}

Dynamical development of information technologies has led to the fact that in the last decades the situation in the field of software and information support has changed significantly. The spread of the Internet has served as an additional incentive for the dissemination of the object approach and the concept of open systems. The problems of cross-platform portability have become topical, a component approach to software design and implementation has spread.

In turn, the modern understanding of the Internet is no longer only as a communication and information environment, but also as a programming environment, presents additional requirements for both software complexes and their developers. Sharply increases the knowledge amount that a modern programmer must possess. The necessary stage of development is the modeling of both data and programs, preferably using special modeling languages and CASE-tools.

Requirements are increasing if it is not just about using separate information technologies: databases, software packages, GIS, expert systems, but also about the integration of heterogeneous technologies within a single software. The problem is complicated if the task is to convert this software into a computational resource accessible via the Internet (especially if you have to deal with legacy software - software packages developed, for example, ten years ago).

The authors have been dealing with these problems for a number of years. The paper presents a methodical approach to the construction of modern software systems, as well as the creation of computing resources accessible via the Internet. The developed technologies are applied to solve the problem of legacy software using the example of the software OPTCON for solving optimal control problems. The presented results obtained in the development of software and information support of scientific research can be useful in many subject areas.

\section{Acknowledgements}

The work is partially supported by Russian Foundation of Basic Research, grant No 18-07-00587.

\section{References}

1. V. A. Baturin, V. I. Gurman, A. I. Moskalenko, Models of natural resources management (Moscow, Nauka, 1981).

2. V. A. Baturin, A. Yu. Gornov and L. V. Massel, Internettechnology of mathematical modeling and computational experiment for solving optimal control problems, Computing technologies, 7 (2002) 339-346.

3. L. V. Massel, E. A. Boldyrev, Modeling and development of modern software systems for energy research, Computing technologies, 7(4) (2002) 59-70.

4. L. V. Massel, A. Yu. Gornov, D. V. Podkamennii Creation of computing resources on the Internet based on legacy software, Computing technologies, 7 (2002) 247252.

5. A. Yu. Gornov, A. O. Divakov, OPTCON software for solving optimal control problems: user guide (Irkutsk, 1993).

6. A. Yu. Gornov, T. S. Zarodnyuk, Tunneling algorithm for solving nonconvex optimal control problems, Optimization, Simulation, and Control, Springer Optimization and Its Applications, 76 (2013) 289-299.

7. A. Yu. Gornov, T. S. Zarodnyuk, E. A. Finkelshtein, A. S. Anikin, The Method of Uniform Monotonous Approximation of the Reachable Set Border for a Controllable System, J Glob Optim, 66(1) (2016) 53-64.

8. V. A. Baturin, D. E. Urbanovich, Approximate methods of optimal control with terminal constraints (Novosibirsk, Nauka, 1997).

9. J. T. Betts, Practical Methods for optimal control using nonlinear programming (Philadelphia, SIAM, 2001).

10. SOCS: User's Guide. Release 7.1 (The Boeing Company, M\&CT-TECH-01-014, www.boeing.com/ assets/pdf/phantom/socs/docs/SOCS_Users_Guide.pdf).

11. Peer-to-peer: harnessing the power of disruptive technologies, Edeted by Andy Oram (ISBN: 0-59600110-X, 2001).

12. A. M. Robachevsky, Operating system UNIX (Saint Petersburg, BHV, 2000). 